\title{
Evaluating Performance Of Wireless Sensor Network In Realistic Smart Grid EnVironment
}

\author{
Laila Nassef $^{1,2}$, Remah Elhebshi ${ }^{1}$ and Linta Jose ${ }^{1}$ \\ ${ }^{1}$ Department of Computer Science, Faculty of Computing and Information Technology, \\ King Abdulaziz University, Jeddah, Saudi Arabia \\ ${ }^{2}$ Department of Computer Sciences and Information, Institute of Statistical Studies and \\ Research, Cairo University, Giza, Egypt
}

\begin{abstract}
Wireless Sensor Networks (WSNs) is a strong candidate for smart grid applications, such as advanced metering infrastructure, demand response management, dynamic pricing, load control, electricity fraud detection, fault diagnostics, substation monitoring and control as well as automation of various elements of the power grid. The realization of these applications directly depends on efficiency of communication facilities among power grid elements. However, the harsh power grid environmental conditions with obstacles, noise, interference, and fading pose great challenges to reliability of these facilities to monitor and control the power grid. The purpose of this paper is to evaluate performance of WSNs in different power grid environments such as $500 \mathrm{kv}$ substations, main power control room, and underground network transformer vaults. The power grid environments are modeled using a log-normal shadowing path loss model channel with realistic parameters. The network is simulated and performance is evaluated using packet delivery ratio, communication delay, and energy consumption. The simulation results have revealed that different environments have considerable impacts on performance of WSNs which make it suitable for most applications that need low data rate with low reliability requirements.
\end{abstract}

\section{KEYWORDS}

Smart Grid; Wireless Sensor Networks; Propagation Models; NS-2

\section{INTRODUCTION}

Smart grid is a new generation of power grid network to modernize the grid using robust two way communications, advanced sensors, and distributed computing technologies to improve the efficiency, reliability and safety of power delivery and use between utility and customer [1]. Electric power grid contains three main subsystems: power generation, power transmission and distribution, and customer facilities [2]. The integration between different parts of the power grid can be achieved using sensing, metering, and communication services provided by Wireless Sensor Network (WSN) [3]. Sensor nodes are small in size and communicate with each other over short distances to provide advanced communication and computing abilities [4]. Each sensor node consists of three sub systems: the sensor subsystem which senses the environment, the processing subsystem which performs local computations on the sensed data and the communication subsystem which is responsible for message exchange with neighbouring sensor nodes. Sensors are deployed on the critical parts of the power grid to enable both utilities and customers to transfer, monitor, predict, and manage energy usage of smart grid effectively and costly [5]. They are widely deployed in various home area networks and field area for monitoring and control applications. The monitored data is either transmitted to a central station or processed locally in a data processing system to provide information about energy consumption condition or 
status of equipment to a remote management system. The monitored data can help to respond to changing conditions and malfunctions of the electric grid components in a proactive manner.

In order to meet the requirements of smart grid in data acquisition, transmission reliability, stability, robustness and low power consumption of devices, WSNs will be used in monitoring and control of various household and/or field deployments of smart grid applications [6]. It supports applications such as pricing at the real time, response according to demand, enhanced support of metering and the control of load [7]. WSNs support various communication protocols for Medium Access Control (MAC) and physical layers. IEEE 802.15.4 standard [8] uses carrier sense multiple access with collision avoidance (CSMA/CA) medium access mechanism. It operates on the unlicensed frequency range of $868 \mathrm{MHz}, 915 \mathrm{MHz}$ and $2.4 \mathrm{GHz}$. It offers a data rate of 20-250 Kbps using Direct Sequence Spread Spectrum (DSSS) technology. It provides a communication range of $10 \mathrm{~m}$ to $100 \mathrm{~m}$ while maintaining significantly low power requirement (1 to $100 \mathrm{~mW}$ ).

Sensors may be subject to radio frequency interference, highly caustic or corrosive environments, high humidity levels, vibrations, dirt and dust, or other conditions that challenge performance [9]. Depending on environment propagation characteristics, an electromagnetic signal may be reflected, scattered, diffracted, diffracted, or absorbed when it propagates [10]. All these effects have two significant effects on the signal strength. First, the signal decreases exponentially with respect to communication distance. Second, for a given communication distance, the signal strength is random and log-normally distributed about the mean distance dependent value. In electric power grid environments, the topology and wireless connectivity of the network may vary due to link failures. These harsh environmental conditions may cause a portion of sensor nodes to malfunction or render the information they gather obsolete [11]. Recent field tests [1] show that smart grid systems have harsh and complex environmental conditions, dynamic topology changes, connectivity problems, variable link qualities and high packet error rates because of interference, fading effects, equipment noise, and obstructions in smart grid environments.

Simulation plays an important role in the verification of WSN's protocols [12]. While the protocols are simulated reasonably realistically, the propagation of wireless transmission channels are not. Simulations typically model propagation with either the free space model or two ray ground model. Such models are only valid in open space where there are no obstructions. The surrounding environments and the presence of obstructions greatly influences propagation and consequently, the open space propagation models are not applicable as they do not represent a real world network propagation model. The characteristics of wireless channels cause fundamental limitations to performance of WSNs and a more realistic model must be used [13]. This calls for comprehensive performance evaluations of communication protocols used in different smart grid environments. Although there are considerable work on WSNs, almost no reference to analyze its performance under harsh power grid environment. Most of the research was based on simplified propagation models, not taking into account realistic propagation models. The impact of various power grid environments on WSNs performance should be evaluated in this real environments. Therefore, the main contribution of this paper is to analyze performance of WSNs in different harsh electric power grid environments using a Log-normal shadowing path loss model with realistic parameters.

The remainder of this paper is organized as follows. Section 2 provides description of WSNs networks. Section 3 explains communication challenges of different smart grid environments. Section 4 describes simulation environment, performance metrics, and simulation scenarios. Results are analyzed in Section 5. Section 6 provides the conclusion and future work. 
International Journal of Wireless \& Mobile Networks (IJWMN) Vol. 10, No. 3, June 2018

\section{WiRELESS SENSOR NETWORKS}

The physical and MAC layers of WSNs are based on IEEE802.15.4 standard [7]. In this type of networks, three types of devices are defined: a coordinator, routers, and end devices. A coordinator is responsible for establishing, maintaining, and controlling all the neighbour devices on the network. It allocates network addresses to other nodes which join the network successively. Routers, which are sometimes called relay nodes, take care of data transmission and have capability to extend the scope of network. End devices collect data and transmit then to routers or coordinators. In addition, network usually contain one or several gateways for communicating with other networks. The coordinator or any of the routers can serve as a gateway [14]. The MAC layer can operate in beacon or non-beacon modes [15]. In beacon enabled mode, the coordinator node will periodically send out a beacon with detailed information about the network and possibly guaranteed time slots for certain nodes using slotted Carrier Sense Multiple Access with Collision Avoidance (CSMA/CA) protocol, along with a superframe structure that is managed by the coordinator. The non beacon enabled mode uses unslotted CSMA-CA as the MAC protocol. The network layer provides dynamic network formation, addressing, routing, and network management functions. It supports three different types of topologies: star topology, peer to peer and cluster tree. Transmission reach and battery life of devices vary depending upon the topology adopted. Three types of topologies are supported: star, peer to peer (mesh), and cluster tree topology. The star topology uses a master-slave network model, where an FFD is chosen to be the coordinator and all other devices in the network can only directly communicate with it. This is different from a mesh topology, where each device is capable of communicating with any other device, as long as they are in radio range with one another. Cluster tree networks are considered a special case of mesh networks in which the majority of nodes are FFDs, and RFDs can connect to the network as leaf nodes. In addition, the RFDs can only communicate with FFDs.

\section{COMmunication Challenges of SMART GRID EnVironments}

WSNs is responsible for identifying, establishing and maintaining routes between transmitter and receiver and facilitating communication when the nodes can no more communicate. Thus, how WSN's perform in the given environment depends on how well it can identify between a good link and bad link during active communication [16]. Fading causes alternating constructive and destructive signal interference at the receiving node. As a result, there is no direct line of sight path and multiple propagated signals are received. This affect the received signal strength, which become the superimposition of direct signal as well as reflected, scattered and diffracted signals [17]. Consequently, the received signal will have a wide varying amplitude and phase, which causes multiple copies that interfere with each other. The interference of two or more multipath signals arriving at the receiver at slightly different times causes multipath fading [18]. This fluctuation in received signal strength may give misleading information about the received signal strength and this could affect performance of the routing protocols in two ways. First, receiver makes a false assumption that the link is no longer usable when it is still usable. This forces the routing protocol to start a new route search resulting into increased consumption of network resources, bandwidth and the battery power of the processing nodes. Second, the receiver assumes a bad link to be a good one and includes it in its route. Thus, during the data transmission, the link fails causing increased network activities through route recovery or additional route discoveries as will be indicated in the paper's results.

Signal propagation in high obstacle environments is unpredictable and its strength fades not only because of distance between sender and receiver but also because of antenna position, transmission power, attenuation due to obstacles. The signal strength depends on signal fluctuation. The short-term fluctuation in the signal amplitude caused by the local multipath is 
called small scale fading. Due to the effect of multipath, a receiver can gets multiple copies of a signal which interfere with each other causes fluctuation is signal strength over a short distance. On the other hand, long term variation in the mean signal level is called large scale fading, also known as shadowing. Variations in the mean signal level are caused by the presence of signal shadow areas due to surrounding elements in the propagation environment. It is characterized by a large distance separating transmitter and receiver. Several statistical models are used to describe fading in wireless environments and the most frequently used distribution for large scale fading is shadowing, while for small scale fading, Rayleigh, and Ricean [19] can be used. These statistical models are used to accurately predict the fading effect. In large scale fading, the shadowing model shows how signal strength fade with distance according to power law and reflect the variation of power at a distance. In small scale fading, a fading in which the reflected signal components reaching the receiver are of almost equal strength is called a Rayleigh fading and the one in which there is one principal component that has higher contribution towards signal reception is called Ricean fading. The instantaneous received power of a given signal may be treated as a stochastic random variable that varies with distance and the selection of a particular model associates a known probability distribution with this random variable.

\subsection{Electrical Power Grid Environments}

The realization of smart grid depends on the communication capabilities of WSNs in harsh and complex electric power grid environments. The environmental noise and interference from nonlinear electric power equipment and fading in harsh smart grid environments, makes reliable communication a challenging task for WSNs for smart grid applications. The nature of propagation channel depends on direct line sight, multipath, presence of obstacles, reflection, scattering, shadowing etc. The received signal is composed of multipath signals with randomly distributed amplitudes and phases, combined to give a resultant signal that varies in time and space [20]. Proper modelling of the power gird environment is needed for proper evaluation of performance of communication facilities in this environments. The power grid environments have been modelled through a comprehensive set of real-world field tests using IEEE 802.15.4 compliant WSNs at Georgia Power, Atlanta, GA, USA [1]. Field tests have been performed to measure noise, channel characteristics, attenuation and link quality of wireless channels in harsh electric power grid environments. Their experimental studies showed that log-normal shadowing path loss model provided more accurate channel models compared to Rayleigh and Nakagami models for wireless environments with obstructions. All their field tests have been conducted over a period of several weeks for various locations and network configurations.

In electrical power grid, the quality of the wireless channel is a complex combination of effects due to path loss, and multipath fading. Path loss quantifies the loss in signal strength due to the distance and the absorption of the objects between two nodes. Shadow fading characterizes the fluctuations around the average path loss. In this model, the distance between transmitter and receiver is not the only variable parameter during simulations. To introduce random events, the shadowing model utilizes a random variable $X$. It requires a reference distance $d_{0}$ to calculate the average received free space signal strength $P_{r, F S}\left(d_{0}\right)$. The path loss exponent $\beta$ in equation (1) depends on the environment and it is constant. Values vary between two (free space) and six (indoor, non-line-of-sight). $X$ is normal distributed with an average of zero and a standard deviation $\sigma$ (called shadow deviation). Again it is non-variable and reasonable values vary between three (factory, line of sight) and twelve (outside of buildings). Values for $\beta$ and $\sigma$ are usually empirically determined. 


$$
\begin{aligned}
& P_{r, S H}(d)=P_{r, F S}\left(d_{0}\right)\left|\frac{d}{d_{0}}\right|^{-\beta} \cdot 10^{X}, \\
& X(x)=\left\{x \varepsilon(-\infty, \infty) \mid \mathrm{P}(\mathrm{x})=\mathrm{N}\left(0, \sigma^{2}\right)\right\}
\end{aligned}
$$

where $P_{r, S H}(d)$ is the average received power, $P_{r, F S}\left(d_{0}\right)$ is average received free space signal strength at a reference distance $\mathrm{d}_{0}$ corresponding to a point located in the far field of the transmit antenna, $\beta$ is the path loss exponent, $X_{\sigma}$ is the zero mean Gaussian random variable with standard deviation $\sigma$. The values of the path loss exponent of the propagation environment $\beta$ and the shadowing deviation $\sigma$ were calculated from the measured data in electric power system environments, using square error of the regression analysis such that the difference between the measured and estimated path losses is minimized in a mean square error sense over a wide range of measurement locations and transmitter receiver separations.

In real smart grid propagation environments, the received signal levels depend on the existence of line of sight (LOS), or the absence of it on the contrary (NLOS). Meanwhile in LOS scenarios the level of direct signal is higher compared to the rest of multipath versions of the propagated signal, in NLOS scenarios there is not a signal component which prevails in terms of amplitude over the rest of them. Six environments correspond to a $500 \mathrm{kV}$ substation (LOS), a $500 \mathrm{kV}$ substation (NLOS), an underground transformer vault (UTV) (LOS), an underground transformer vault (NLOS), a main power room (MPR) (LOS) and a main power room (NLOS). The seventh environment represents a non-smart grid environment. The log normal shadowing model is used for modelling the wireless links of various power grid environments radio propagation parameters for these different electric power environments are summarized in Table 1[1].

\section{Simulation EnVironMent}

To investigate the effects of harsh smart grid environments on performance of WSNs, a network consisting of 15 sensor nodes is simulated on Network Simulator (NS-2) [21]. These nodes are configured in a star topology over an area of 50x50 square meter area. The star topology is used between the nodes and the coordinator to simulate application scenarios where sensor nodes reporting monitoring data to a central base station or gateway. The traffic is Constant Bit Rate (CBR) that is used to generate eight traffic flows. Each CBR connection started at random period of time. Once a CBR connection started, it continued generating packet till the end of the simulation. The packet generation rate varies from 1 packet per second to 5 packets per second. Each simulation was tested for an arbitrary 100 seconds simulation time. To statistically analyze performance, 10 runs are used to measure the average values of three performance metrics: packet delivery ratio, communication delay and energy consumption.

The packet delivery ratio is the ratio between the number of successful packets and the total number of transmitted packets. Packet loss may occur at any stage of a network transmission, mainly due to link failures and CSMA/CA channel access mechanism. It is an important metric which can be used as an indicator to a congested network. Communication delay is the average time to receive all data on the destination side. It is the sum of delays at all links within the endto-end path including processing delay, queuing delay, transmission delay, propagation delay and retransmission delay. Energy consumption is the average percentage of the consumed energy in network measured in Joules. The common simulation parameters are presented in Table 2.

The simulations are conducted in seven scenarios in electric power grid environments. The log normal shadowing model is used based on experimentally determined log normal channel parameters for different smart grid environments. Six scenarios correspond to environments of a $500 \mathrm{kV}$ substation (LOS), a $500 \mathrm{kV}$ substation (NLOS), an underground transformer vault (LOS), 
an underground transformer vault (NLOS), a main power room (LOS) and a main power room (NLOS). The seventh scenario represents the non smart grid environment.

Table 1: Log Normal Shadowing Channel Parameters

\begin{tabular}{|l|c|c|}
\hline Propagation Environment & $\begin{array}{c}\text { Path Loss } \\
\boldsymbol{\beta}\end{array}$ & $\begin{array}{c}\text { Shadowing deviation } \\
\boldsymbol{\sigma}\end{array}$ \\
\hline $\begin{array}{l}\text { 500-kv substation (LOS) } \\
\text { outdoor }\end{array}$ & 2.42 & 3.12 \\
\hline $\begin{array}{l}\text { 500-kv substation (NLOS) } \\
\text { Outdoor }\end{array}$ & 3.51 & 2.95 \\
\hline Underground network transformer vault (LOS) & 1.45 & 2.54 \\
\hline Underground network transformer vault (NLOS) & 3.15 & 3.19 \\
\hline $\begin{array}{l}\text { Main power room(LOS) } \\
\text { Indoor }\end{array}$ & 1.64 & 3.29 \\
\hline $\begin{array}{l}\text { Main power room(NLOS) } \\
\text { Indoor }\end{array}$ & 2.38 & 2.25 \\
\hline $\begin{array}{l}\text { Non smart grid environment } \\
\text { Indoor environment }\end{array}$ & 1.4 & 4 \\
\hline $\begin{array}{l}\text { 500-kv substation (LOS) } \\
\text { Outdoor }\end{array}$ & 2.42 & 3.12 \\
\hline $\begin{array}{l}\text { 500-kv substation (NLOS) } \\
\text { Outdoor }\end{array}$ & 3.51 & 2.95 \\
\hline Underground network transformer vault (LOS) & 1.45 & 2.54 \\
\hline Underground network transformer vault (NLOS) & 3.15 & 3.19 \\
\hline $\begin{array}{l}\text { Main power room(LOS) } \\
\text { Indoor }\end{array}$ & 1.64 & 3.29 \\
\hline $\begin{array}{l}\text { Main power room(NLOS) } \\
\text { Indoor }\end{array}$ & 2.38 & 2.25 \\
\hline $\begin{array}{l}\text { Non smart grid environment } \\
\text { Indoor environment }\end{array}$ & 1.4 & 4 \\
\hline
\end{tabular}

\section{Simulation RESUlts}

Figure 1 shows the packet delivery ratio in different smart grid environments as a function of data rates. At lower data rates, packet delivery ratio is $92 \%$ because of legitimate packets loss. As higher data rate is applied to the network, more and more packets are dropped due to collisions and bad link quality in harsh smart grid environments. The signal attenuation caused by obstacles in NLOS environment have lowered the packet delivery ratio. Packets have to wait for longer period of time due to unsuccessful packet receptions causing delivery ratio to decrease and consequently to increase communication delay in Figure 2. At lower data rates, the MPR environment has lower delivery ratio compared to the UTV and $500 \mathrm{kV}$ environments because higher value of path loss exponent which cause higher signal attenuation and lower the probability of receiving packets. As data rates increases, the MPR-NLOS gives the lowest delivery ratio because packets are dropped due to collisions and bad link quality. This would increase the number of retransmissions, effectively congesting the network even further. The inconsistent values at data rate of 4 packets per second for MPR-NLOS and $500 \mathrm{kv}$-LOS are due to link quality variations of wireless links in harsh power grid environments. The non smart grid environment gives the highest delivery ratio.

Figure 2 shows the communication delay as a function of data rates. As data rate increases, the communication delay increases because of high packet loss, congestion and wireless channel characteristics in harsh smart grid environments. The non smart grid environment produced highest delay compared with six smart grid environments at data rate of 3 packets / second. At lower data rates, the NLOS environments exhibit less delay than the LOS environments which 
International Journal of Wireless \& Mobile Networks (IJWMN) Vol. 10, No. 3, June 2018

have higher number of retransmissions. At higher data rates, UTV environment shows better delay than $500 \mathrm{kv}$ and MPR environments.

Table 2: Common Simulation Parameters

\begin{tabular}{|l|l|}
\hline Simulation Parameter & Value \\
\hline Network topology & Star \\
\hline Simulation Time & 100 Seconds \\
\hline Number of nodes & 15 \\
\hline Packet length & 70 bytes \\
\hline Packet Type & Constant bit rate \\
\hline Propagation Model & Shadowing Model \\
\hline Queue Type & Drop tail \\
\hline Number of traffic flows & 8 \\
\hline MAC Protocol & IEEE 802.15 .4 \\
\hline Terrain Size & $50 \mathrm{X} 50 \mathrm{~m}^{2}$ \\
\hline Data rate & $1-5$ packets $/$ second \\
\hline
\end{tabular}

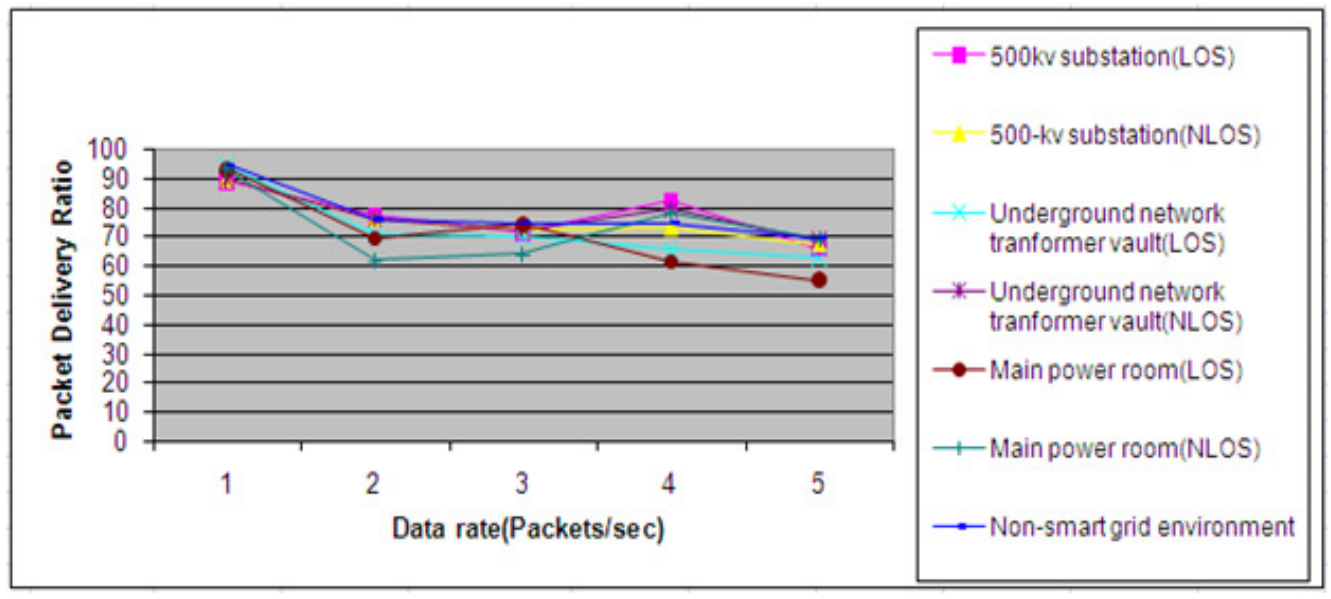

Figure 1: Packet Delivery Ratio versus Data Rates

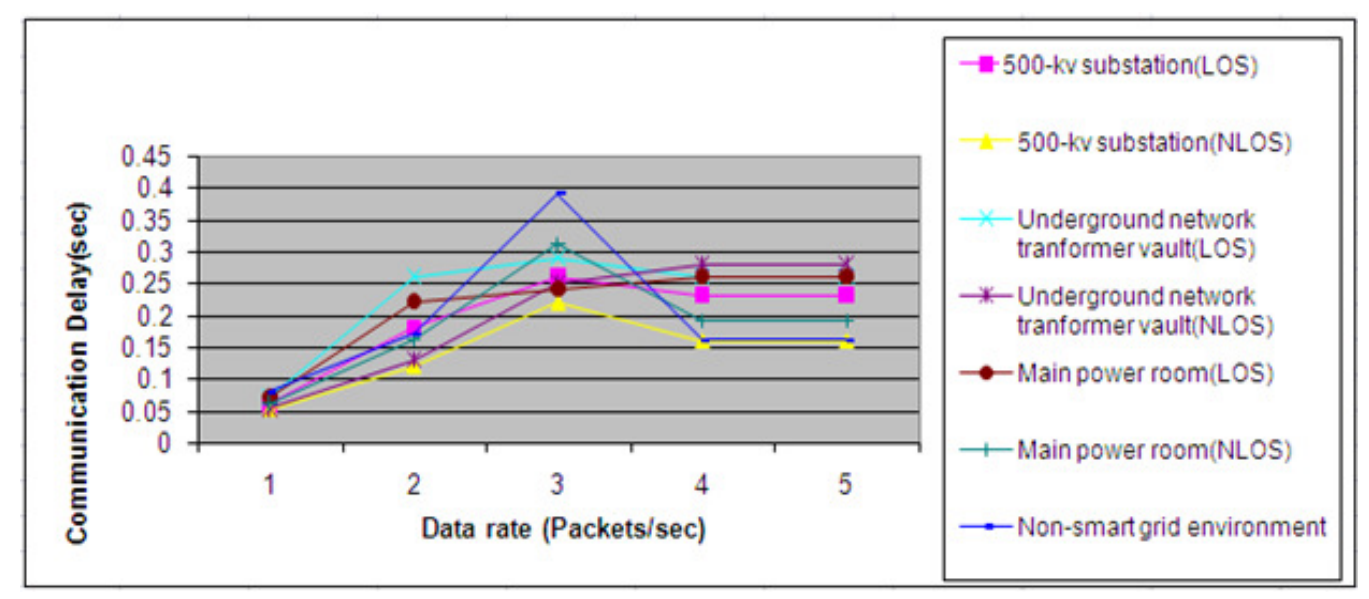

Figure 2: Communication Delay versus Data Rates 
International Journal of Wireless \& Mobile Networks (IJWMN) Vol. 10, No. 3, June 2018

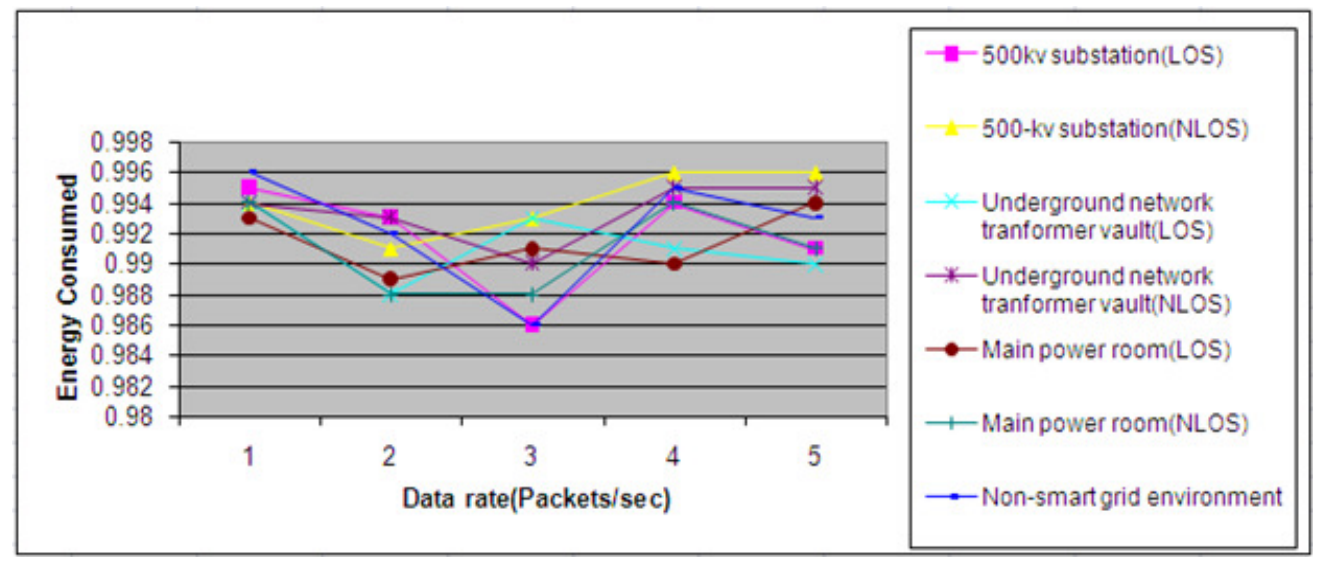

Figure 3: Energy Consumption versus Data Rates

Figure 3 shows the energy consumed in different smart grid environments as a function of data rates. At lower data rate, the energy consumption decreases with the increase of data rate, because of direct proportionality of the energy consumption and packet delivery ratio in Figure 1. The sudden drop in energy consumption for data rate of $3 \mathrm{Pkts} / \mathrm{sec}$ is attributed to the high increase in delay in Figure 2 due to network congestion. At higher data rates, energy consumption increases because of retransmissions due high packet loss, congestion and wireless channel characteristics in harsh smart grid environments. The energy consumption of NLOS environments is relatively higher than the LOS environments because of high number of retransmissions and packet drop of the harsh NLOS environments. At lower data rates, the MPR environment has consumed less energy compared to the $500 \mathrm{kV}$ and UTV environments. At higher data rates, the 500kv-NLOS consumed the highest energy.

\section{CONCLuSion ANd Future Work}

The probabilistic propagation models can have great impact on performance of WSNs. Therefore, more attention must be paid to study characteristics of the realistic environments. This paper presented a simulation study to analyze the effects of different smart grid environments on the performance of WSNs for smart grid applications. The motivation is to determine how the realistic channel characteristics would affect performance metrics. This paper investigated performance of WSNs in different harsh conditions of power grid environments in terms of shadowing deviation and pass loss exponents parameters. The simulation results have revealed that different environments of power grid have considerable impact on performance of WSNs. The higher path loss exponents of different power grid environments have resulted in higher signal attenuation and reduced the probability of receiving packets which significantly degraded the performance of WSNs under high traffic loads. The LOS environment generally produced better performance, while the worst results were for NLOS environments due to big variations in the received signal. The overall results showed that IEEE802.15.4 based WSNs can only be used in low data rate and low power applications with low reliability requirements. The different power grid environments have affected performance and there is a need to develop an adaptable and reliable protocols that takes into account interference awareness and link quality characteristics for different smart grid environments. A cross layer design and optimization techniques must be applied to improve performance of WSNs in harsh smart grid environments. 
International Journal of Wireless \& Mobile Networks (IJWMN) Vol. 10, No. 3, June 2018

\section{REFERENCES}

[1] S. Bi, C. K. Ho \& R. Zhang, (08 April 2015) "Wireless powered communication: opportunities and challenges," IEEE Communications Magazine, Vol. 53, Issue: 4, pp. 117 - 125, DOI: 10.1109/MCOM.2015.7081084.

[2] A. Leonardi, K. Mathioudakis, A. Wiesmaier \& F. Zeiger, (2014) "Towards the Smart Grid: Substation Automation Architecture and Technologies," Hindawi Publishing Corporation, Vol. 2014, Article ID 896296, http://dx.doi.org/10.1155/2014/896296.

[3] M. Erol-Kantarci \& H. Mouftah, (17 March 2011) "Wireless Sensor Networks for Cost-Efficient Residential Energy Management in the Smart Grid," IEEE Transactions on Smart Grid, Vol. 2, Issue: 2, pp. 314 - 325, DOI: 10.1109/TSG.2011.2114678.

[4] I. Dietrich \& F. Dressler, (February 2009) "On the lifetime of wireless sensor networks," ACM Transactions on Sensor Networks (TOSN), Vol. 5 Issue: 1, pp. 1872 - 1899, DOI: $10.1145 / 1464420.1464425$.

[5] B. Rashid \& H. Rehmani, (January 2016) "Applications of wireless sensor networks for urban areas: A survey," Journal of Network and Computer Applications, Vol. 60, pp. 192-219, https://doi.org/10.1016/j.jnca.2015.09.008.

[6] C. Greer, D. Wollman, D. Prochaska \& P. Boynton, (October 01, 2014) "NIST Framework and Roadmap or Smart Grid interoperability Standards, Release 1.0," NIST Standards.

[7] "http://www.ZigBee.org/Standards/ZigBeeSmartEnergy/Overview.aspx," Zigbee Smart Energy Profile. [Online].

[8] "http://www.ieee802.org/," IEEE802. [Online].

[9] H. S. Savitha, K. S. Divya, Abhilasha, Chandhini \& S. Manasa, (May 2017) "Industrial Wireless Sensor Networks: Challenges,Design Principles, and Technical Approaches," International Journal of Engineering Research in Electronics and Communication Engineering (IJERECE), pp. 215-221, ISSN (Online) 2394-6849.

[10] L. L.Nassef, (December 2010) "On the Effects of Fading and Mobility in On Demand Routing Protocols," Egyptian Informatics Journal, Vol. 11, Issue 2, pp. 67-74, http://dx.doi.org/10.1016/j.eij.2010.10.003.

[11] P. Sharma \& G. Pandove, (May-June 2017) "A Review Article on Wireless Sensor Network in Smart Grid," International Journal of Advanced Research in Computer Science, Vol. 8, No. 5, pp. 19031907, ISSN No. 0976-5697.

[12] X. Zhang and W. Li, (2014) "Simulation of the smart grid communications: Challenges, techniques, and future trends," Computers and Electrical Engineering, Vol. 40, pp. 270-288, http://dx.doi.org/10.1016/j.compeleceng.2013.11.022.

[13] S. Jabbar, M. A. Habib, A. A. Minhas, M. Ahmad, R. Ashraf, S. Khalid \& K. Han, (18 February 2018) "Analysis of Factors Affecting Energy Aware Routing in Wireless Sensor Network," Wireless Communications and Mobile Computing- Hindawi, Vol. 2018, Article ID 9087269, pp. 396-405, https://doi.org/10.1155/2018/9087269.

[14] S. Jabbar, M. Asif Habib, A. Minhas, M. Ahmad, R. Ashraf, S. Khalid \& K. Han, (2018) "Analysis of Factors Affecting Energy Aware Routing in Wireless Sensor Network," Hindawi, Wireless Communications and Mobile Computing, Vol. 2018, Article ID 9087269, https://doi.org/10.1155/2018/9087269. 
International Journal of Wireless \& Mobile Networks (IJWMN) Vol. 10, No. 3, June 2018

[15] B. Kim, H. Park, K. H. Kim, D. Godfrey \& K.-I. Kim, (2017) "A Survey on Real-Time Communications in Wireless Sensor Networks," Hindawi, Wireless Communications and Mobile Computing, Vol. 2017, Article ID 1864847, https://doi.org/10.1155/2017/1864847.

[16] M. Doddavenkatappa, M. Choon Chan \& B. Leong, (03 January 2012) "Improving Link Quality by Exploiting Channel Diversity in Wireless Sensor Networks," in Real-Time Systems Symposium (RTSS), 2011 IEEE 32nd, Vienna, Austria, DOI: 10.1109/RTSS.2011.22.

[17] F. Alassery, (November 2017) "A virtual MIMO transmission scenarios for high energy efficiency smart wireless sensor networks over Rayleigh flat fading channel," in Information Technology, Electronics and Mobile Communication Conference (IEMCON), 2017 8th IEEE Annual, Vancouver, BC, Canada, 23 DOI: 10.1109/IEMCON.2017.8117127.

[18] H. Kim, (07 January 2016) "An energy-efficient load balancing scheme to extend lifetime in wireless sensor networks," Springer US, Vol. 19, Issue 1, pp. 279-283, https://doi.org/10.1007/s10586-0150526-9.

[19] Y. Dong, M. Hossain \& J. Cheng, (11 February 2016) "Performance of Wireless Powered Amplify and Forward Relaying Over Nakagami-m Fading Channels With Nonlinear Energy Harvester," IEEE Communications Letters, Vol. 20, Issue 4, pp. 672 - 675, DOI: 10.1109/LCOMM.2016.2528260.

[20] W. Chongburee \& O. Musikanon , (2012) "ZigBee Propagations and Performance Analysis in Last Mile Network," International Journal of Innovation, Management and Technology, Vol. 3, No. 4.

[21] "http://www.isi.edu/nsnam/ns/," The Network Simulator, NS-2. [Online]. 